\title{
Platonic sphalerons in Yang-Mills-Higgs-dilaton theory
}

\author{
Burkhard Kleihaus, Jutta Kunz, Kari Myklevoll* \\ Insitute of Physics, University of Oldenburg, Germany \\ E-mail: kari.myklevoll@uni-oldenburg.de, \\ kleihaus@theorie.physik. uni-oldenburg.de, \\ kunz@theorie.physik.uni-oldenburg.de
}

\begin{abstract}
Electroweak sphalerons, first described by Klinkhamer and Manton, are unstable static classical solutions related to baryon-number-violating processes. Electroweak sphaleron solutions with spherical and axial symmetries have been known for some time. Recently, also sphaleron solutions without rotational symmetry have been found. These sphaleron solutions have the symmetries of crystals or platonic bodies. We therefore call them platonic sphalerons. They have similarities to platonic skyrmions and platonic monopoles found earlier. We here present platonic sphaleron solutions in SU(2) Yang-Mills-Higgs theory coupled to a scalar dilaton field. The dilaton field mimics the effect of gravitation concerning the existence and behaviour of classical solutions. We therefore regard these solutions as a first step towards obtaining self-gravitating static solutions without rotational symmetries, and in particular black holes with only discrete symmetries.
\end{abstract}

International Europhysics Conference on High Energy Physics

July 21st - 27th 2005

Lisboa, Portugal

${ }^{*}$ Speaker. 


\section{Introduction}

Non-linear field theories in flat spacetime often possess static, localized solutions with finite energy. Famous examples are the monopoles and sphalerons of SU(2) Yang-Mills-Higgs (YMH) theory. Monopoles are predicted by Grand Unified Theories, while sphalerons arise already in the electroweak sector of the Standard Model. Both are relevant in particle physics with implications for cosmology [1].

More recently it became clear, that solitons are also important in string theory, where gravity is essential. Whereas most of these gravitating solutions involve Abelian fields, non-Abelian solutions may play an important role as well [2]. But the low energy effective action of string theory also involves a dilaton field, and likewise the dimensionally reduced action of supergravity theories. From this point of view one would like to study solitons of YMH theory coupled to a dilaton and to gravity.

The spatial symmetries of static classical solutions can be specified by means of certain rational maps of degree $N$ [3]. For monopoles the degree $N$ is associated with the topological charge, for sphalerons with the Chern-Simons number. When $N \geq 3$, solutions with only discrete symmetries appear $[4,5]$. Identifying these with the symmetries of platonic solids or crystals, we refer to them as platonic monopoles or platonic sphalerons. However, so far little is known about such static solutions with only discrete symmetries, whereas static solutions with rotational symmetry are well studied, both from a mathematical and a physical point of view.

The central question we would like to address is whether such solutions with only discrete symmetries exist also in the presence of gravity. The existence of globally regular static solutions with no rotational symmetries would represent a completely new type of gravitating solutions.

In view of the complexity of the coupled Einstein-Yang-Mills-Higgs (EYMH) equations in the absence of rotational symmetry [6], we here turn to a simplified theory, where the influence of gravity is mimicked by the presence of a dilaton [7], since it is known, that the coupling of gravity to YMH theory has a similar effect concerning the existence and the properties of static classical solutions with rotational symmetry as the coupling to a dilaton.

\section{Yang-Mills-Higgs-dilaton theory}

We consider static finite energy solutions of Yang-Mills-Higgs-dilaton (YMHD) theory, with SU(2) gauge potential $V_{0}=0, V_{i}=V_{i}(\vec{r}), i=1,2,3$, Higgs doublet $\Phi=\Phi(\vec{r})$, and dilaton field $\phi=\phi(\vec{r})$. The energy of such solutions is given by

$$
\frac{4 \pi v}{g} E=\int\left(\frac{1}{2} \partial_{i} \phi \partial^{i} \phi+\frac{1}{2} e^{2 \kappa \phi} \operatorname{Tr}\left(F_{i j} F^{i j}\right)+\left(D_{i} \Phi\right)^{\dagger}\left(D^{i} \Phi\right)+\lambda e^{-2 \kappa \phi}\left(\Phi^{\dagger} \Phi-\frac{v^{2}}{2}\right)^{2}\right) d^{3} r,
$$

with field strength tensor $F_{i j}=\partial_{i} V_{j}-\partial_{j} V_{i}+i g\left[V_{i}, V_{j}\right]$, gauge potential $V_{i}=V_{i}^{a} \tau_{a} / 2$, and covariant derivative of the Higgs field $D_{i} \Phi=\partial_{i} \Phi+i g V_{i} \Phi$. Here $g$ and $\kappa$ denote the gauge and dilaton coupling constants, respectively, $\lambda$ denotes the strength of the Higgs self-interaction, and $v / \sqrt{2}$ the vacuum expectation value of the Higgs field. In the limit of vanishing dilaton coupling constant, the model corresponds to the bosonic sector of Weinberg-Salam theory for vanishing Weinberg angle. The energy $E$ is related to the dilaton charge $D=\alpha g /(4 \pi) \int_{S_{2}} \vec{\nabla} \phi \cdot d \vec{S}$, by the simple relation $D=\alpha^{2} E$, where $\alpha=\kappa v$. 

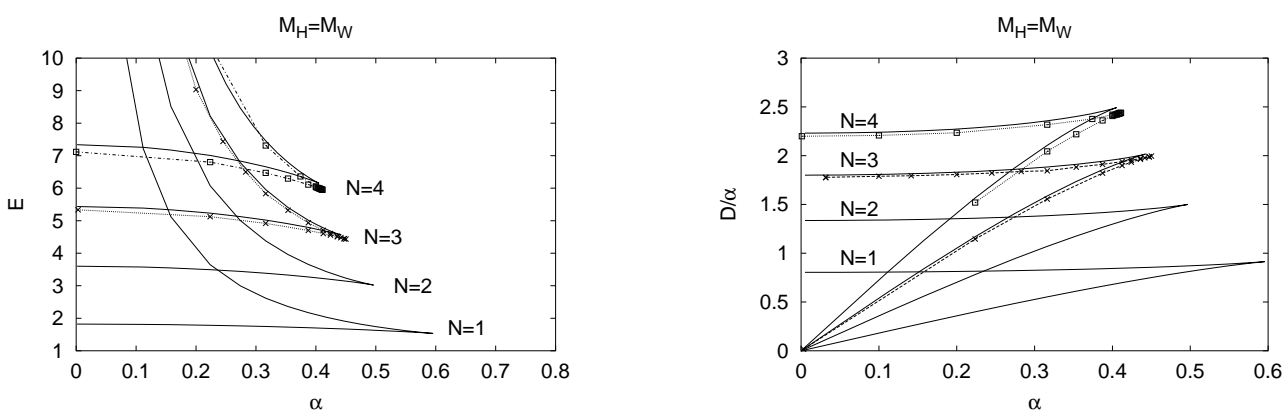

Figure 1: The energy $E$ (left) and scaled energy $\alpha E$ (right) of the spherically symmetric $(N=1)$, axially symmetric $(N=2-4)$ and platonic sphalerons $(N=3,4$, represented by symbols) is shown as a function of the dilaton coupling $\alpha$, for $M_{H}=M_{W}$, where $M_{W}=g v / 2$.

\section{Platonic sphaleron solutions}

Platonic YMHD sphalerons involve nine gauge field functions, three Higgs field functions, and a dilaton function. The numerical solutions are constructed with help of the software package FIDISOL [8]. Here we consider sphalerons with spherical $(N=1)$, axial $(N=2,3,4)$, tetrahedral $(N=3)$ and cubic $(N=4)$ symmetry.

To obtain solutions with discrete symmetry we make use of rational maps. A rational map of degree $N$ is a function $R: S^{2} \mapsto S^{2}$, where $R(\xi)=p(\xi) / q(\xi), p$ and $q$ are polynomials of degree at most $N$, and the complex coordinate $\xi$ on the sphere can be identified with conventional polar coordinates by $\xi=\tan (\theta / 2) e^{i \varphi}[3]$.

We here consider platonic YMHD solutions associated with the maps $R_{N}$ [3],

$$
R_{3}(\xi)=\frac{\sqrt{3} i \xi^{2}-1}{\xi\left(\xi^{2}-\sqrt{3} i\right)}, \quad R_{4}(\xi)=\frac{\xi^{4}+2 \sqrt{3} i \xi^{2}+1}{\xi^{4}-2 \sqrt{3} i \xi^{2}+1}
$$

For fundamental platonic sphalerons, the boundary conditions of the fields at spatial infinity are given by the rational map, which thereby specifies the symmetry of the solution. For the first excited platonic sphalerons, in contrast, the rational map (and the discrete symmetry) enters through the start configuration for the numerical algorithm [9].

When considering the energy of the sphaleron solutions as a function of the dimensionless dilaton coupling constant $\alpha$, we observe that for each value of $N$ and Higgs mass $M_{H}=v \sqrt{2 \lambda}$, there are two branches of solutions, merging at a maximal value of $\alpha$. On the lower branch in the limit of vanishing $\alpha$, the fundamental YMHD sphalerons approach smoothly the corresponding sphalerons of Weinberg-Salam theory. On the upper branch the energy diverges in the limit $\alpha \rightarrow 0$, but the scaled energy $\alpha E$ approaches a finite limiting value. Indeed, after a scale transformation, the scaled solutions on the upper branch approach sphaleron solutions of Yang-Mills-dilaton (YMD) theory. In figure 1 we show the energy $E$ (left) and the scaled energy $\alpha E=D / \alpha$ (right), of fundamental sphalerons as functions of $\alpha$ for the Higgs mass $M_{H}=M_{W}$. Currently we are also constructing the fundamental and first excited $N=4$ platonic sphalerons of YMD theory [9].

In figure 2 we exhibit surfaces of constant energy density of the fundamental YMHD sphalerons with tetrahedral and cubic symmetry, close to their respective maximal values of $\alpha$. 

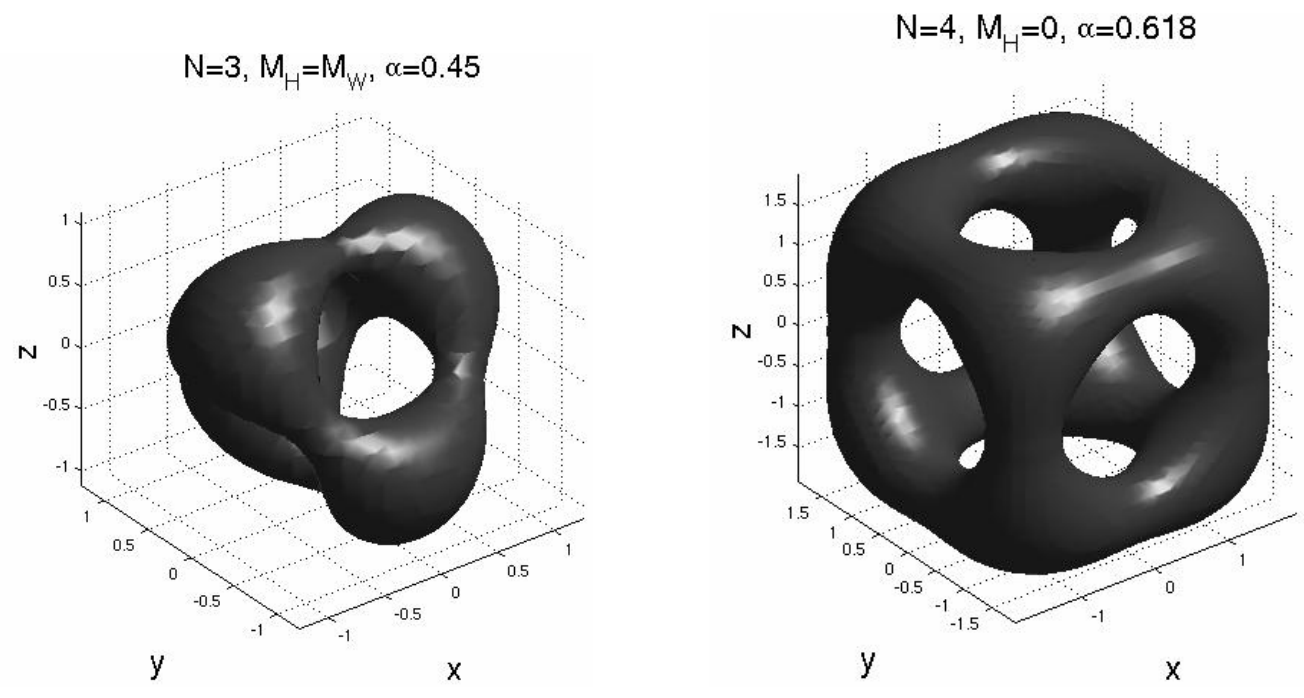

Figure 2: The dimensionless energy density $\varepsilon$ of platonic sphalerons with $N=3$ and 4 .

\section{Summary and outlook}

Motivated by the aim to demonstrate the existence of non-perturbative gravitating solutions with only discrete symmetries, we have constructed platonic solutions in the presence of a dilaton. While studying the effects of a dilaton is interesting in itself, since dilatons arise naturally in higher dimensional theories, we have here included the dilaton mainly to mimick the effects of gravity.

In general, the existence of gravitating (static) regular solutions involving non-Abelian fields, is related to the existence of black holes with non-Abelian hair [2]. Therefore the conjectured existence of regular solutions with platonic symmetries strongly indicates the existence of a completely new type of black holes: static black holes with only discrete symmetries [6].

\section{References}

[1] see e.g. E. W. Kolb, M. S. Turner, The Early Universe, Addison-Wesley, 1990.

[2] M. S. Volkov and D. V. Gal'tsov, Phys. Rept. 319 (1999) 1.

[3] C. J. Houghton, N. S. Manton and P. M. Sutcliffe, Nucl. Phys. B 510 (1998) 507.

[4] P. M. Sutcliffe, Int. J. Mod. Phys. A 12 (1997) 4663.

[5] B. Kleihaus, J. Kunz, and K. Myklevoll, Phys. Lett. $B 582$ (2004) 187.

[6] S. A. Ridgway, and E. J. Weinberg, Gen. Rel. Grav. 27 (1995) 1017; Phys. Rev. D51 (1995) 638; Phys. Rev. D52 (1995) 3440.

[7] B. Kleihaus, J. Kunz and K. Myklevoll, Phys. Lett. $\mathbf{B 6 0 5}$ (2005) 151.

[8] W. Schönauer and R. Weiß, J. Comput. Appl. Math. 27 (1989) 279; M. Schauder, R. Weiß and W. Schönauer, The CADSOL Program Package, Universität Karlsruhe, Interner Bericht Nr. 46/92 (1992).

[9] B. Kleihaus, J. Kunz, and K. Myklevoll, Phys. Lett. B632 (2006) 333; Particle-like platonic solutions in scalar gravity, submitted to Phys. Lett. B, hep-th/0601124. 